\title{
RESOURCE BOUNDED IMMUNITY AND SIMPLICITY *
}

\author{
Extended Abstract
}

\author{
Toshio Suzuki $^{1}$ and Tomoyuki Yamakami ${ }^{2}$ \\ ${ }^{1}$ Dept. of Math, and Inform. Sci., Osaka Prefecture University, Osaka, 599-8531 Japan \\ ${ }^{2}$ Computer Science Program, Trent University, Peterborough, Ontario, Canada K9J 7B8
}

\begin{abstract}
Revisiting the thirty years-old notions of resource-bounded immunity and simplicity, we investigate the structural characteristics of various immunity notions: strong immunity, almost immunity, and hyperimmunity as well as their corresponding simplicity notions. We also study $\boldsymbol{k}$-immunity and $\boldsymbol{k}$-simplicity and their extensions: feasible $\boldsymbol{k}$-immunity and feasible $\boldsymbol{k}$-simplicity. Finally, we propose the $\boldsymbol{k}$-immune hypothesis as a working hypothesis that ensures the existence of simple sets in NP.
\end{abstract}

Keywords: immune set, simple set, complete set, forcing, generic oracle, random oracle

\section{Foundations of Immunity and Simplicity}

The original notions of immunity and simplicity date back to mid 1940s. Post [17] first constructed a simple set for the class of recursively enumerable sets. The new breed of resource-bounded immunity and simplicity waited to be introduced until mid 1970s by an early work of Flajolet and Steyaert [5]. In their seminal paper, Flajolet and Steyaert constructed various recursive sets that, for instance, have no infinite DTIME $(t(n))$-subsets under the term "DTIME( $(\boldsymbol{n}(\boldsymbol{n}))$-immune sets." Later, Ko and Moore [11] studied the polynomial-time bounded immunity, which is now preferably called P-immune sets. Subsequently, Balcázar and Schöning [2] considered Pbi-immune sets, which are P-immune sets whose complements are also P-immune. Homer and Maass [8] extensively discussed the cousin of P-immune sets, known as NP-simple sets. The importance of these notions was widely recognized in 1980s. Since these notions can be easily expanded to any complexity class $\mathcal{C}$, we begin with

\footnotetext{
* This work was in part supported by the Natural Sciences and Engineering Research Council ofCanada and Grant-in-Aid for Scientific Research (No. 14740082), Japan Ministry of Education, Culture, Sports, Science, and Technology. This work was done while the first author visited the University of Ottawa between September and December of 2000.
} 
an introduction of the general notions of $\mathcal{C}$-immune sets, $\mathcal{C}$-bi-immune sets, and $\mathcal{C}$ simple sets. These notions are further expanded in various manners in later sections.

DEFINITION 1 Let $\mathcal{C}$ be any complexity class of languages over alphabet $\Sigma$.

1 A set $S$ is $\mathcal{C}$-immune if $S$ is infinite and there is no infinite subset of $S$ in $\mathcal{C}$.

2 A set $S$ is $\mathcal{C}$-bi-immune if $S$ and $\bar{S}$ are both $\mathcal{C}$-immune.

3 A set $S$ is $\mathcal{C}$-simple if $S$ belongs to $\mathcal{C}$ and $\overline{\boldsymbol{S}}$ is $\mathcal{C}$-immune.

Note that the existence of a $\mathcal{C}$-simple set immediately implies $\mathcal{C} \neq$ co- $\mathcal{C}$; however, the separation $\mathcal{C} \neq$ co- $\mathcal{C}$ does not necessarily guarantee the existence of $\mathcal{C}$-simple sets.

Throughout this paper, we set our alphabet $\Sigma$ to be $\{0,1\}$. Let $\mathbb{N}$ (or $\boldsymbol{\omega}$ ) denote the set of all nonnegative integers and set $\mathbb{N}^{+}=\mathbb{N}-\{\mathbf{0}\}$. All logarithms are taken to base 2 and a polynomial means a multivariate polynomial with integer coefficients. We assume a standard bijection from $\Sigma^{<\omega}$ to $\Sigma^{*}$ that is polynomial-time computable and polynomial-time invertible, where $\Sigma^{<\omega}$ is the set of all finite sequences of strings over $\boldsymbol{\Sigma}$. This bijection enables us to identify $\Sigma^{<\omega}$ with $\boldsymbol{\Sigma}^{*}$. We use multi-tape off-line Turing machines (TMs, in short) as a model of computation. Assumed is the reader's familiarity with basic complexity classes, such as P, NP, E (linear exponential time), and EXP (polynomial exponential time). This paper focuses mostly on the complexity classes lying in the polynomial-time hierarchy ${ }^{1}\left\{\Delta_{k}^{\mathrm{P}}, \Sigma_{k}^{\mathrm{P}}, \Pi_{k}^{\mathrm{P}} \mid k \in \mathbb{N}\right\}$ [12].

We mainly use "partial" functions and all functions are presumed to be singlevalued. Since total functions are also partial functions, we will define function classes as collections of partial functions and, whenever we need total functions, we will explicitly indicate the totality of functions. Now, fix $k \in \mathbb{N}^{+}$. The notation $\mathbf{F} \Delta_{k}^{\mathrm{P}}$ denotes the collection of all single-valued partial functions $f$ such that there exist a set $B \in \Sigma_{k-1}^{\mathrm{P}}$ and a polynomial-time deterministic oracle TM $M$ satisfying the following condition: for every $x$, if $x \in \operatorname{dom}(f)$ then $M^{B}(x)$ halts in an accepting state and outputs $f(x)$ and otherwise, $M^{B}(x)$ halts in a rejecting state (in this case, $f(x)$ is undefined). In particular, write FP for $\mathrm{F} \Delta_{1}^{\mathrm{P}}$.

A set $A$ is called $\Delta_{k}^{\mathrm{P}}$-m-reducible to another set $B$ via a reduction $f$ if $f$ is a total $\mathrm{F} \Delta_{k}^{\mathrm{P}}$-function from $\Sigma^{*}$ to $\Sigma^{*}$ and $A=\{x \mid f(x) \in B\}$. If in addition $f$ is honest ${ }^{2}$, then we say that $A$ is $\boldsymbol{h}$ - $\Delta_{k}^{\mathrm{P}}$-m-reducible to $B$. Moreover, a set $A$ is $\Delta_{k}^{\mathrm{P}}$-tt-reducible to $B$ via a reduction triplet $(\boldsymbol{\nu}, f, \alpha)$ if(i) $\nu$ is a total $\mathrm{F} \Delta_{k}^{\mathrm{P}}$-function from $\Sigma^{*}$ to $\{0\}^{*}$, (ii) $f$ is a total $\mathrm{F} \Delta_{k}^{\mathrm{P}}$-function from $\Sigma^{*}$ to $\Sigma^{*}$ such that, for every $x, f(x)=\left\langle y_{1}, y_{2}, \ldots, y_{k}\right\rangle$ for certain strings $y_{1}, y_{2}, \ldots, y_{k}$, where $k=|\nu(x)|$, (iii) $\alpha$ is a total $\mathrm{F} \Delta_{k}^{\mathrm{P}}$-function from $\Sigma^{*} \times \Sigma^{*}$ to $\{0,1\}$ such that $A=\{x \mid \alpha(x, B(f(x)))=1\}$, where $B(f(x))$ is an abbreviation of the $k$-bit string $B\left(y_{1}\right) B\left(y_{2}\right) \cdots B\left(y_{k}\right)$ when $f(x)=\left\langle y_{1}, \ldots, y_{k}\right\rangle$. If in addition $f$ is componentwise honest ${ }^{3}$, then $A$ is $h-\Delta_{k}^{P}$-tt-reducible to $B$. The notion of completeness can be induced from its corresponding reducibility.

\footnotetext{
${ }^{1}$ The polynomial-time hierarchy consists of the classes defined in the following fashion: $\Delta_{0}^{\mathrm{P}}=\Sigma_{0}^{\mathrm{P}}=$ $\Pi_{0}^{\mathrm{P}}=\mathrm{P}, \Sigma_{k+1}^{\mathrm{P}}=\mathrm{NP} \Sigma_{k}^{\mathrm{P}}$, and $\Pi_{k+1}^{\mathrm{P}}=\operatorname{co}-\Sigma_{k+1}^{\mathrm{P}}$ for any number $k \in \mathbb{N}$.

${ }^{2}$ A partial function $\boldsymbol{f}$ from $\boldsymbol{\Sigma}^{*}$ to $\boldsymbol{\Sigma}^{*}$ is polynomially honest (honest, for short) if there exists a polynomial $p$ such that $|x| \leq p(|f(x)|)$ for all strings $x \in \operatorname{dom}(f)$.

${ }^{3}$ A partial function from $\boldsymbol{\Sigma}^{*}$ to $\boldsymbol{\Sigma}^{<\boldsymbol{\omega}}$ (which is identified with $\boldsymbol{\Sigma}^{*}$ ) is componentwise honest if there exists a polynomial $\boldsymbol{p}$ such that, for every $x \in \operatorname{dom}(f),|x| \leq p\left(\left|y_{i}\right|\right)$ for all $i \in\{1,2, \ldots, k\}$, provided that $f(x)=\left\langle y_{1}, y_{2}, \ldots, y_{k}\right\rangle$.
} 
It is well-known that P-immune sets exist even in the class E. In particular, Ko and Moore [11] constructed a P-immune set that is also P-tt-complete for E. Note that no h-P-m-complete set for NP can be P-immune since the image of a P-immune set by a polynomial-time computable reduction is either finite or P-immune. Using a relativization technique, Bennett and Gill [3] showed that a P-immune set exists in NP relative to a random oracle with probability 1 . A recursive oracle relative to which NP contains P-immune sets was later constructed by Homer and Maass [8]. Torenvliet and van Emde Boas [22] strengthened their results by demonstrating a relativized world where NP has a P-immune set which is also NP-simple.

The notion of $\mathcal{C}$-immunity is closely related to various other notions, which include complexity cores [13] and instance complexity [15]. We can naturally expand these characterizations to more general $\Sigma_{k}^{\mathrm{P}}$-immune and $\Delta_{k}^{\mathrm{P}}$-immune sets. Balcázar and Schöning [2] also built a bridge between P-bi-immune sets and finite-to-one reductions. Expanding their argument, we give in Lemma 2 a characterization of $\mathcal{C}$-biimmunity as well as $\mathcal{C}$-immunity.

For any partial function $f$ from $\Sigma^{*}$ to $\Sigma^{*}$, the set $G r a p h(f)=\{\langle x, f(x)\rangle \mid x \in$ $\operatorname{dom}(f)\}$ is called the graph of $f$. Let $\Sigma_{0}^{\mathrm{PSV}}=$ FP and let $\Sigma_{k}^{\mathrm{P} S V}$ denote the class of all single-valued partial functions $f$ such that $f$ is polynomially bounded ${ }^{4}$ and $\operatorname{Graph}(f)$ is in $\Sigma_{k}^{\mathrm{P}}$. For brevity, we write NPSV for $\Sigma_{1}^{\mathrm{P} S V}$. For any $k \in \mathbb{N}$ and any $A, B \subseteq \Sigma^{*}$, a single-valued partial function $f$ from $\Sigma^{*}$ to $\Sigma^{*}$ is called a $\Sigma_{k}^{P}$ m-quasireduction ( $\Delta_{k}^{P}$-m-quasireduction, resp.) from $A$ to $B$ if (i) $f$ is in $\Sigma_{k}^{P} S V$ (F $\Delta_{k}^{P}$, resp.), (ii) $\operatorname{dom}(f)$ is infinite, and (iii) for any string $x \in \operatorname{dom}(f), x \in A$ iff $f(x) \in B$. For any string $u \in \Sigma^{*}$, the inverse image $f^{-1}(u)$ of $f$ at $u$ is the set $\{x \in \operatorname{dom}(f) \mid f(x)=u\}$. Notice that $f^{-1}(u)=\emptyset$ if $u \notin \operatorname{ran}(f)$.

\section{LEMMA 2 Let $\mathcal{C} \in\left\{\Delta_{k}^{\mathrm{P}}, \Sigma_{k}^{\mathrm{P}} \mid k \in \mathbb{N}\right\}$ and $S \subseteq \Sigma^{*}$.}

$1 S$ is $\mathcal{C}$-immune if and only if (i) $S$ is infinite and (ii) for every set $B$, every $\mathcal{C}$-m-quasireduction from $S$ to $B$, and every $u$ in $B, f^{-1}(u)$ is finite.

$2 S$ is $\mathcal{C}$-bi-immune if and only if (i) $S$ is infinite and (ii) for every set $B$, every $\mathcal{C}$-m-quasireduction from $S$ to $B$, and every $u$ in $\Sigma^{*}, f^{-1}(u)$ is finite.

The characterization given in Lemma 2(2) led Balcázar and Schöning [2] to introduce a stronger notion of P-bi-immunity: strong P-bi-immunity. A more general notion, called strong $\mathcal{C}$-immunity, will be introduced in Section 2.

Whether an NP-simple set exists is one of the long-standing open problems because such a set separates NP from co-NP. Nonetheless, NP-simple sets are known to exist in various relativized worlds. In early 1980s, Homer and Maass [8] and Balcázar [1] constructed relativized worlds where an NP-simple set exists. Later, Vereshchagin [24] proved that, relative to a random oracle, an NP-simple set exists with probability 1. From Theorem 9 in Section 2, for instance, it immediately follows that an NPsimple set exists relative to a generic oracle. Torenvliet [21] built an oracle relative to which a $\Sigma_{2}^{\mathrm{P}}$-simple set exists. For a much higher level $k$ of the polynomial-time

\footnotetext{
${ }^{4}$ A partial function $\boldsymbol{f}$ from $\boldsymbol{\Sigma}^{*}$ to $\boldsymbol{\Sigma}^{*}$ is polynomially bounded if there exists a polynomial $\boldsymbol{p}$ such that $|f(x)| \leq p(|x|)$ for any string $x \in \operatorname{dom}(f)$.
} 
hierarchy, Bruschi [4] constructed an oracle relative to which $\boldsymbol{\Sigma}_{\boldsymbol{k}}^{\mathbf{P}}$-simple sets exist using the size lower bounds of certain nonuniform constant-depth circuits.

In the rest of this section, we focus on closure properties of the class of all $\Sigma_{k}^{\mathrm{P}}$ immune sets because no such closure property has been systematically studied in the literature. A complexity class $\mathcal{C}$ is said to be closed downward under a reduction $\leq_{r}$ on infinite sets if, for any pair of infinite sets $A$ and $B, A \leq_{r} B$ and $B \in \mathcal{C}$ imply $A \in \mathcal{C}$. Here, we study three reducibilities. Let $k \in \mathbb{N}$. A set $A$ is $\Delta_{k}^{\mathrm{P}}$-d-reducible to $B$ via $f$ if $f$ is a total $\mathrm{F} \Delta_{k}^{\mathrm{P}}$-function from $\Sigma^{*}$ to $\Sigma^{<\omega}$ (which is identified with $\boldsymbol{\Sigma}^{*}$ ) and $A=\{x \mid B \cap \operatorname{set}(f(x)) \neq \emptyset\}$, where $\operatorname{set}\left(\left\langle y_{1}, y_{2}, \ldots, y_{m}\right\rangle\right)=\left\{y_{1}, y_{2}, \ldots, y_{m}\right\}$. By contrast, $A$ is $\Delta_{k}^{\mathbf{P}}$-c-reducible to $B$ via $f$ if $\bar{A}$ is $\Delta_{k}^{\mathbf{P}}$-d-reducible to $\bar{B}$ via $f$. For any fixed $i \in \mathbb{N}^{+}, A$ is $\Delta_{k}^{\mathrm{P}}$-itt-reducible to $B$ via $(f, \alpha)$ if $A$ is $\Delta_{k}^{\mathrm{P}}$-tt-reducible to $B$ via $(\nu, f, \alpha)$, where $\nu(x)=0^{i}$ for any $x$. For any reductibility $r$ using computation $\mathcal{C}$, we say that $A$ is $h$-C-r-reducible to $B$ if $A$ is $\mathcal{C}$-r-reducible to $B$ via $f$ (or $(f, \alpha)$ )such that $f$ is componentwise honest.

Now, we claim that the class of all $\Sigma_{k}^{P}$-immune sets is closed downward under $\mathrm{h}-\Delta_{k}^{\mathrm{P}}$-c-reductions on infinite sets; however, we cannot replace this conjunctive reducibility by disjunctive reducibility.

\section{THEOREM 3 Let $k \in \mathbb{N}^{+}$.}

1 The class of all $\Sigma_{k}^{\mathrm{P}}$-immune sets is closed downward under $h-\Delta_{k}^{\mathrm{P}}-c$-reductions on infinite sets.

2 The class of all NP-immune sets is not closed under $h$-P-d-reductions or $h-\mathrm{P}$ 2 tt-reductions on infinite sets.

The first claim of Theorem 3 is easy and is shown as follows. Assume that an infinite set $A$ is $\mathbf{h}-\Delta_{k}^{\mathbf{P}}$-c-reducible to a $\boldsymbol{\Sigma}_{k}^{\mathrm{P}}$-immune set $B$ via a componentwise-honest reduction $f$. If $A$ contains an infinite $\Sigma_{k}^{P}$-subset $C$, then consider the set $D=\{y \mid$ $\exists x \in C[|x| \leq p(|y|) \wedge y \in \operatorname{set}(f(x))]\}$, where $p$ is a polynomial such that $|x| \leq$ $p(|y|)$ for all $\boldsymbol{x}$ and all $\boldsymbol{y} \in \boldsymbol{s e t}(f(\boldsymbol{x}))$. Clearly, $D$ is an infinite $\boldsymbol{\Sigma}_{\boldsymbol{k}}^{P}$-subset of $B$, a contradiction. Therefore, $A$ is $\Sigma_{k}^{\mathrm{P}}$-immune.

How complex are $\boldsymbol{\Sigma}_{k}^{\mathrm{P}}$-simple sets? Intuitively, $\mathcal{C}$-simple sets are "thin" and thus cannot be "complete" for the class $\mathcal{C}$. As an immediate consequence of Theorem 3(1), we obtain the following corollary.

COROLLARY 4 Let $k \in \mathbb{N}^{+}$. No $\Sigma_{k}^{\mathrm{P}}$-simple set is $\boldsymbol{h}-\Delta_{k}^{\mathrm{P}}$-d-complete for $\boldsymbol{\Sigma}_{k}^{\mathrm{P}}$.

Recently, Agrawal (cited in [19]) showed, using the NP-levelability of $\overline{\text { SAT }}$ (assuming SAT $\notin \mathrm{P}$ ), that no NP-simple set is h-P-btt-complete for NP, where SAT is the set of all satisfiable quantifier-free Boolean formulas. His argument will be generalized in Section 4 in connection to $\mathcal{C}$-hyperimmune sets.

\section{Strong Immunity and Strong Simplicity}

Following the introduction of P-bi-immunity, Balcázar and Schöning [2] stepped forward to introduce the notion of strongly P-bi-immunity, which comes from the quasireducibility-characterization of P-bi-immunity given in Lemma 2(2). While Pbi-immunity requires its quasireductions to be finite-to-one, strong P-bi-immunity requires the quasireductions to be almost one-to-one, where a quasireduction $f$ is called 
almost one-to-one on a set $S$ if the collision set $\left\{(x, y) \in(\operatorname{dom}(f) \cap S)^{2} \mid x<\right.$ $y \wedge f(x)=f(y)\}$ is finite. Such strongly P-bi-immune sets are known to exist even in the class $\mathrm{E}$ [2].

Generalizing the notion of P-bi-immunity, we can introduce strong $\mathcal{C}$-bi-immunity for any complexity class $\mathcal{C}$ lying in the polynomial-time hierarchy. Moreover, we newly introduce the notions of strong $\mathcal{C}$-immunity and strong $\mathcal{C}$-simplicity. Recall that $\Sigma_{k}^{\mathrm{P}}$-m-quasireductions are all single-valued functions in $\Sigma_{k}^{\mathrm{P} S V}$ for each $k \in \mathbb{N}^{+}$.

Definition 5 Let $\mathcal{C} \in\left\{\Delta_{k}^{P}, \Sigma_{k}^{P} \mid k \in \mathbb{N}\right\}$.

1 A set $S$ is strongly $\mathcal{C}$-immune if (i) $S$ is infinite and (ii) for every set $B$ and every $\mathcal{C}$-m-quasireduction from $S$ to $B, f$ is almost one-to-one on $S$.

$2 A$ set $S$ is strongly $\mathcal{C}$-bi-immune if $S$ and $\overline{\boldsymbol{S}}$ are both strongly $\mathcal{C}$-immune.

3 A set $S$ is strongly $\mathcal{C}$-simple if $S$ is in $\mathcal{C}$ and $\overline{\boldsymbol{S}}$ is strongly $\mathcal{C}$-immune.

In particular, when $\mathcal{C}=\mathrm{P}$, Definition 5(2) coincides with the notion of P-biimmunity originally given in [2].

LEMMA 6 For any complexity class $\mathcal{C} \in\left\{\Delta_{k}^{\mathrm{P}}, \boldsymbol{\Sigma}_{k}^{\mathrm{P}} \mid k \in \mathbb{N}\right\}$, every strongly $\mathcal{C}$ immune set is $\mathcal{C}$-immune and every strongly $\mathcal{C}$-simple set is $\mathcal{C}$-simple.

A major difference between $\mathcal{C}$-immunity and strong $\mathcal{C}$-immunity is shown in the following example. For any NP-immune set $A$, the disjoint union ${ }^{5} \boldsymbol{A} \oplus \boldsymbol{A}$ is also NPimmune; on the contrary, it is not strongly NP-immune because $A \oplus A$ can be reduced to $A$ by the almost two-to-one function $f$ defined as $f(\lambda)=\lambda$ and $f(x b)=x$ for $b \in$ $\{0,1\}$, where $\lambda$ is the empty string. Therefore, the class of all strongly NP-immune sets is not closed under the disjoint-union operator. Historically, using the structural difference between these two notions, Balcázar and Schöning [2] constructed a set in E which is P-bi-immune but not strongly P-bi-immune.

We show a closure property of the class of strongly $\Sigma_{k}^{\mathrm{P}}$-immune sets. If $A$ is $\Delta_{k}^{\mathrm{P}}-\mathrm{m}$ reducible to $B$ via a one-to-one honest reduction $f$, we say that $A$ is $h-\Delta_{k}^{\mathrm{P}}-1$-reducible to $B$ via $f$.

\section{PROPOSITION 7 Let $k \in \mathbb{N}^{+}$.}

1 The class of all strongly $\Sigma_{k}^{\mathrm{P}}$-immune sets is closed downward under $\boldsymbol{h}-\Delta_{k}^{\mathrm{P}}-1$ reductions on infinite sets.

2 The class of all strongly NP-immune sets is not closed downward under h-P$m$-reductions on infinite sets.

COROLlaRY 8 For each level $k \in \mathbb{N}^{+}$, there is no strongly $\boldsymbol{\Sigma}_{k}^{\mathrm{P}}$-simple set that is $h-\Delta_{k}^{\mathrm{P}}$-1-complete for $\boldsymbol{\Sigma}_{k}^{\mathrm{P}}$.

Finally, we turn our interest to relativization. For each $k \in \mathbb{N}^{+}$, it is easy to show that a strongly $\Sigma_{k}^{P}$-simple set exists relative to a recursive oracle (similar to Proposition

\footnotetext{
${ }^{5}$ The disjoint union of $A$ and $B$ is the set $\{0 x \mid x \in A\} \cup\{1 x \mid x \in B\}$.
} 
26). Even relative to a random oracle, there exists a strongly NP-simple set with probability 1 (similar to Proposition 27). Employing weak forcing, we now prove the following relativization result.

THEOREM 9 A strongly $\mathrm{NP}^{G}$-simple set exists relative to a generic oracle $G$.

\section{Almost Immunity and Almost Simplicity}

We have shown in the previous section that strong $\mathcal{C}$-immunity and its simplicity strengthen the ordinary notion of $\mathcal{C}$-immunity and $\mathcal{C}$-simplicity. In contrast to these notions, Orponen [14] and Orponen, Russo, and Schöning [16] expanded P-immunity to the new notion of almost P-immunity. The complementary notion of almost Pimmunity under the term P-levelability (a more general term "levelable sets" was first used by Ko [10] in a resource-bounded setting) was extensively discussed by Orponen et al. [16]. Naturally, we can generalize these notions to almost $\mathcal{C}$-immunity and $\mathcal{C}$ levelability for any complexity class $\mathcal{C}$. Furthermore, we newly introduce the notion of almost $\mathcal{C}$-bi-immunity and almost $\mathcal{C}$-simplicity.

DEFINITION 10 Let $\mathcal{C}$ be any complexity class.

1 A set $S$ is almost $\mathcal{C}$-immune if $S$ is the union of a $\mathcal{C}$-immune set and a set in $\mathcal{C}$.

2 An infinite set is $\mathcal{C}$-levelable if it is not almost $\mathcal{C}$-immune.

$3 \mathrm{~A}$ set $S$ is almost $\mathcal{C}$-bi-immune if $S$ and $\overline{\boldsymbol{S}}$ are both almost $\mathcal{C}$-immune.

$4 A$ set $S$ is almost $\mathcal{C}$-simple if $S$ is an infinite set in $\mathcal{C}$ and $\overline{\boldsymbol{S}}$ is the union of a set $A$ in $\mathcal{C}$ and a $\mathcal{C}$-immune set $B$, where the difference $B \backslash A$ is infinite.

It follows from Definition 10(1) that every almost $\mathcal{C}$-immune set is infinite since so is every $\mathcal{C}$-immune set. The definition of almost $\mathcal{C}$-simplicity in Definition 10(4) is slightly different from other simplicity definitions because the infinity condition of the difference $B \backslash A$ is necessary to guarantee $\mathcal{C} \neq$ co- $\mathcal{C}$, provided that an almost $\mathcal{C}$-simple set exists.

LEMMA 11 Let $\mathcal{C}$ be any complexity class closed under finite variations, finite union and finite intersection. If an almost $\mathcal{C}$-simple set exists, then $\mathcal{C} \neq$ co- $\mathcal{C}$.

Lemma 11 is shown as follows. Take any set $S$ such that $\bar{S}=A \cup B$ for a set $A \in \mathcal{C}$ and a $\mathcal{C}$-immune set $B$. Suppose $\mathcal{C}=$ co- $\mathcal{C}$. Note that $B \backslash A \subseteq B$ and $B \backslash A=\bar{S} \backslash A \in \mathcal{C}$. Since $B$ is $\mathcal{C}$-immune, $B \backslash A$ must be finite.

The following lemma is immediate from Definition 10.

LemMa 12 For any complexity class $\mathcal{C}$, every $\mathcal{C}$-immune set is almost $\mathcal{C}$-immune and every $\mathcal{C}$-simple set is almost $\mathcal{C}$-simple.

Several characterizations of almost P-immunity and P-levelability are shown in [16] in terms of maximal P-subsets and P-to-finite reductions. We can naturally expand these characterizations to almost $\Delta_{k}^{\mathrm{P}}$-immunity and $\Delta_{k}^{\mathrm{P}}$-levelability (but not to the $\boldsymbol{\Sigma}$-level classes of the polynomial-time hierarchy). 
To understand the characteristics of almost $\mathcal{C}$-immunity, we begin with a simple observation. It is known in [16] that any honestly paddable ${ }^{6}$ set not in $\mathrm{P}$ is P-levelable. As observed in [18], the essence of this assertion is that if $\boldsymbol{A} \notin \mathbf{P}$ and $A$ is lengthincreasing $\mathrm{P}$-m-selfreducible then $A$ is $\mathrm{P}$-levelable, where $A$ is length-increasing $\mathcal{C}$ m-selfreducible if $A$ is $\mathcal{C}$-m-reducible to $A$ via a certain length-increasing reduction. This observation can be generalized to $\Delta_{k}^{\mathrm{P}}$-levelable sets in the following lemma.

LEMMA 13 Let $\boldsymbol{k} \in \mathbb{N}^{+}$and $A \subseteq \Sigma^{*}$. Assuming that $A \notin \Delta_{k}^{\mathrm{P}}$, if $A$ is lengthincreasing $\Delta_{k}^{\mathrm{P}}$-m-selfreducible, then $A$ and $\bar{A}$ are both $\Delta_{k}^{\mathrm{P}}$-levelable. Thus, if $\Delta_{k}^{\mathrm{P}} \neq$ $\boldsymbol{\Sigma}_{k}^{\mathbf{P}}$ then $\boldsymbol{\Sigma}_{\boldsymbol{k}}^{\mathbf{P}}$ as well as $\boldsymbol{\Pi}_{\boldsymbol{k}}^{\mathbf{P}}$ has a $\Delta_{k}^{\mathbf{P}}$-levelable set.

Most known NP-m-complete sets are known to be honestly paddable and thus, by Lemma 13, the complements of these sets are P-levelable sets, which are also NPlevelable. Therefore, most known NP-m-complete sets cannot be almost NP-simple. This result can be compared to Proposition 16.

Now, we assume a standard effective enumeration $\left\{\varphi_{s}\right\}_{s \in \Sigma^{*}}$ of all nondeterministic TMs $\varphi_{s}$. For each index $s$, define the set $W_{s}=\left\{x \mid \varphi_{s}(x) \downarrow=1\right\}$, where " $\varphi_{s}(x) \downarrow$ " means that $\varphi_{s}$ eventually halts on input $x$. Fix $k \in \mathbb{N}$. Let $\mathbf{N P}_{(k)}$ denote the collection of all sets $W_{s}$ such that, for any string $x \in W_{s}$, the running time of $\varphi_{s}$ on input $x$ is at most $|s| \cdot|x|^{k}+|s|$. Moreover, we set $I N D E X_{(k)}=\left\{s \mid W_{s} \in N_{(k)}\right\}$. Note that $\mathrm{NP}=\bigcup_{k \in \mathbf{N}} \mathbf{N P}(k)$.

Earlier, Ko and Moore [11] considered the resource-bounded notion of "productive sets." Another formulation based on $\mathbf{N P}_{(\boldsymbol{k})}$ was later given by Joseph and Young [9], who used the terminology of $k$-creative ${ }^{7}$ sets, where $k$ is any number in $\mathbb{N}^{+}$. They showed that every $\boldsymbol{k}$-creative set is P-m-complete for NP. Orponen et al. [16] showed that, unless $\mathrm{P}=\mathrm{NP}$, every honestly $\boldsymbol{k}$-creative set is $\mathrm{P}$-levelable by demonstrating that any honestly $\boldsymbol{k}$-creative set is length-increasing P-m-selfreducible. From Lemma 13 , it follows that any honestly $k$-creative set and its complement are both P-levelable. Consequently, we obtain the following result.

COROLlARY 14 For any $\boldsymbol{k} \in \mathbb{N}^{+}$, no honestly $\boldsymbol{k}$-creative set is almost NP-simple.

Our notion of almost $\mathcal{C}$-simplicity is similar to what Uspenskii [23] discussed under the term "pseudosimplicity." Here, we give a resource-bounded version of his pseudosimplicity. A set $S$ is called $\mathcal{C}$-pseudosimple if there is an infinite $\mathcal{C}$-subset $A$ of $\overline{\boldsymbol{S}}$ such that $\boldsymbol{S} \cup \boldsymbol{A}$ is $\mathcal{C}$-simple. Although $\mathcal{C}$-simple sets cannot be $\mathcal{C}$-pseudosimple by our definition, any infinite $\mathcal{C}$-pseudosimple set is almost $\mathcal{C}$-simple. The latter claim is shown as follows. Suppose that $S$ is an infinite $\mathcal{C}$-pseudosimple set and $A$ is a $\mathcal{C}$-subset of $\bar{S}$ for which $S \cup A$ is $\mathcal{C}$-simple. This means that $\bar{S} \backslash A$ is $\mathcal{C}$-immune. Therefore, $S$ is almost $\mathcal{C}$-simple.

\footnotetext{
${ }^{6} \mathrm{~A}$ set $S$ is polynomially paddable (paddable, in short) if there is an one-to-one total FP-function pad (called the padding function) from $\Sigma^{*}$ to $\Sigma^{*}$ such that, for all pairs $(\boldsymbol{x}, \boldsymbol{y}) \in \boldsymbol{\Sigma}^{*} \times \boldsymbol{\Sigma}^{*}, \boldsymbol{x} \in \boldsymbol{S}$ iff $\boldsymbol{p a d}(\langle\boldsymbol{x}, \boldsymbol{y}\rangle) \in \boldsymbol{S}$. A set $S$ is honestlypaddable if it is paddable with a padding function that is componentwise honest.

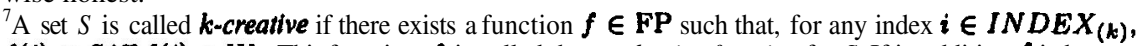
$f(i) \in S$ iff $f(i) \in W_{\boldsymbol{i}}$. This function $\boldsymbol{f}$ is called the productive function for $S$. If in addition $\boldsymbol{f}$ is honest, $S$ is called honestly $\boldsymbol{k}$-creative.
} 
The following theorem shows a close connection among simplicity, almost simplicity, and pseudosimplicity.

THEOREM 15 For each $k \in \mathbb{N}^{+}$, the following three statements are equivalent.

1 There exists a $\Sigma_{k}^{\mathrm{P}}$-simple set.

2 There exists an infinite $\Sigma_{k}^{\mathrm{P}}$-pseudosimple set in $\mathrm{P}$.

3 There exists an almost $\Sigma_{k}^{\mathrm{P}}$-simple set in $\mathrm{P}$.

The most essential part of Theorem 15 is the implication from 1 to 2 . Assume that $S$ is an almost $\Sigma_{k}^{\mathrm{P}}$-simple set. Let $A_{1}=1 S$ and $B_{1}=1 \bar{S}$. Clearly, $B_{1}$ is infinite. Note that $A_{1}$ is a $\Sigma_{k}^{\mathrm{P}}$-subset of $1 \Sigma^{*}$. Since $\bar{S}$ is $\Sigma_{k}^{\mathrm{P}}$-immune and $B_{1} \subseteq 1 \bar{S}, B_{1}$ is $\Sigma_{k}^{\mathrm{P}}$-immune. Since $B_{1}=1 \Sigma^{*} \cap \overline{A_{1}}$, the set $0 \Sigma^{*} \cup A_{1}$ is $\Sigma_{k}^{\mathrm{P}}$-simple. Hence, $0 \Sigma^{*}$ is $\Sigma_{k}^{\mathrm{P}}$-pseudosimple, as required. Similarly, $1 \Sigma^{*}$ is $\Sigma_{k}^{\mathrm{P}}$-pseudosimple.

Theorem 15 indicates the importance of the structure of $\mathrm{P}$ in the course of the study of $\Sigma_{k}^{P}$-simplicity. In a relativized world where a $\Sigma_{k}^{P}$-simple set exists [4], since Theorem 15 relativizes, there exists an almost $\Sigma_{k}^{P}$-simple set within P.

Finally, we briefly discuss a closure property of the class of all almost $\Sigma_{k}^{\mathrm{P}}$-immune sets under polynomial-time reductions. For each number $k \in \mathbb{N}^{+}$, the class of all almost $\boldsymbol{\Sigma}_{k}^{\mathrm{P}}$-immune sets is closed under $\mathrm{h}-\Delta_{k}^{\mathrm{P}}-\mathrm{m}$-reductions on infinite sets. This immediately implies the following consequence.

PROPOSITION 16 For each number $k \in \mathbb{N}^{+}$, there is no almost $\Sigma_{k}^{\mathrm{P}}$-simple set that is $h-\Delta_{k}^{\mathrm{P}}-m$-completefor $\mathbf{\Sigma}_{k}^{\mathrm{P}}$.

\section{Hyperimmunity and Hypersimplicity}

Since Post [17] constructed a so-called hypersimple set, the notions of hyperimmunity and hypersimplicity have played a significant role in the progress of classical recursion theory. A resource-bounded version of these notions was first considered by Yamakami [26] and studied extensively by Schaefer and Fenner [19]. The definition of Schaefer and Fenner is based on the notion of "honest NP-arrays", which differs from the notion of "strong arrays" in recursion theory, where a strong array is a series of pairwise disjoint finite sets. For our formalization, we demand only "eventually disjointness" for sets in an array rather than "pairwise disjointness."

A binary string $x$ is said to represent a finite set $\left\{a_{1}, a_{2}, \ldots, a_{k}\right\}$ if and only if $x=\left\langle a_{1}, a_{2}, \ldots, a_{k}\right\rangle$ and $a_{1}<a_{2}<\cdots<a_{k}$ in the lexicographic order on $\Sigma^{*}$. For convenience, we say that a set $A$ surpasses another set $B$ if there exists a string $z \in A$ satisfying $z>x$ (lexicographically) for all strings $x$ in $B$.

DEFINITION 17 Let $k \in \mathbb{N}^{+}, A \subseteq \Sigma^{*}$, and $\mathcal{C} \in\left\{\Sigma_{k}^{\mathrm{P}}, \Delta_{k}^{\mathrm{P}}\right\}$.

1 An infinite sequence $\mathcal{D}=\left\{D_{s}\right\}_{s \in \Sigma^{*}}$ of finite sets is called a $\Sigma_{k}^{\mathrm{P}}$-array $\left(\Delta_{k}^{\mathrm{P}}\right.$ array, resp.) if there exists a single-valued partial function $f$ in $\Sigma_{k}^{\mathbf{P} S V} \mathbf{F} \Delta_{k}^{\mathbf{P}}$, resp.) such that (i) $\operatorname{dom}(f)$ is infinite, (ii) $D_{s} \neq \emptyset$ and $f(s)$ represents $D_{s}$ for any string $s \in \operatorname{dom}(f)$, and (iii) $D_{s}=\emptyset$ for any string $s \notin \operatorname{dom}(f)$. This $f$ is called the supporting function of $\mathcal{D}$ and the set $\bigcup_{s \in d o m(f)} D_{s}$ is called the support of $\mathcal{D}$. The width of $\mathcal{D}$ is the supremum of the cardinality $\left|D_{\boldsymbol{s}}\right|$ over all $s \in \operatorname{dom}(f)$. 
$2 A \mathcal{C}$-array $\mathcal{D}$ has an infinite support if the support of $\mathcal{D}$ is infinite.

3 A $\mathcal{C}$-array $\left\{D_{s}\right\}_{s \in \Sigma^{*}}$ via $f$ is polynomially honest (honest, in short) if $f$ is componentwise honest; namely, there exists a polynomial $\boldsymbol{p}$ satisfying that $|\boldsymbol{s}| \leq$ $p(|x|)$ for any $s \in \operatorname{dom}(f)$ and any $x \in D_{\mathbf{s}}$.

$4 A \mathcal{C}$-array $\left\{D_{\boldsymbol{s}}\right\}_{\boldsymbol{s} \in \Sigma^{*}}$ via $\boldsymbol{f}$ is eventually disjoint if, for every string $\boldsymbol{x}$ in $\operatorname{dom}(f)$, there exists a string $\boldsymbol{y}$ in $\operatorname{dom}(f)$ such that $\boldsymbol{y} \geq x$ (lexicographically), $D_{\boldsymbol{y}}$ surpasses $D_{x}$, and $D_{x} \cap D_{y}=\emptyset$.

$5 A \mathcal{C}$-array $\left\{D_{s}\right\}_{s \in \Sigma^{*}}$ via $f$ intersects $A$ if $D_{s} \cap A \neq \emptyset$ for all $s \in \operatorname{dom}(f)$.

The honesty condition of an $\mathcal{C}$-array guarantees that the array is eventually disjoint. In addition, any eventually-disjoint $\mathcal{C}$-array has an infinite support because, for any element $D$ in the array, we can always find another disjoint element $D^{\prime}$.

A simple relationship between $\boldsymbol{\Sigma}_{k}^{P}$-simplicity and a honest $\boldsymbol{\Sigma}_{k}^{P}$-array is given in the following lemma, which was implicitly proven by Yamakami [26] and later explicitly stated in [19] for the case where $k=1$.

LEMMA 18 Let $k \in \mathbb{N}^{+}$and let $A$ be any $\Sigma_{k}^{P}$-simple set. For every number $\ell \in \mathbb{N}^{+}$, there is no honest $\boldsymbol{\Sigma}_{\boldsymbol{k}}^{\mathbb{P}}$-array $\mathcal{D}$ such that (i) the width of $\mathcal{D}$ is at most $\boldsymbol{\ell}$ and (ii) $\mathcal{D}$ intersects $A$.

We introduce below the notions of $\mathcal{C}$-hyperimmunity and honest $\mathcal{C}$-hyperimmunity.

DEFINITION 19 Let $\mathcal{C} \in\left\{\Delta_{k}^{\mathrm{P}}, \Sigma_{k}^{\mathrm{P}} \mid k \in \mathbb{N}\right\}$.

1 A set $S$ is (honestly) $\mathcal{C}$-hyperimmune if $S$ is infinite and there is no (honest) $\mathcal{C}$-array $\mathcal{D}$ such that $\mathcal{D}$ is eventually disjoint and $\mathcal{D}$ intersects $A$.

2 A set $S$ is (honestly) $\mathcal{C}$-bi-hyperimmune if $S$ and $\overline{\boldsymbol{S}}$ are both (honestly) $\mathcal{C}$ hyperimmune.

3 A set $S$ is (honestly) $\mathcal{C}$-hypersimple if $\boldsymbol{S} \in \mathcal{C}$ and $\overline{\boldsymbol{S}}$ is (honestly) $\mathcal{C}$-hyperimmune.

Note that "NP-hyperimmunity" defined by Schaefer and Fenner [19] coincides with our honest NP-hyperimmunity. The following relationship between immunity and hyperimmunity can be obtained immediately from Definition 19.

LEMMA 20 For any complexity class $\mathcal{C} \in\left\{\Sigma_{k}^{\mathrm{P}}, \Delta_{k}^{\mathrm{P}} \mid k \in \mathbb{N}\right\}$, every honestly $\mathcal{C}$ hyperimmune set is $\mathcal{C}$-immune and every honestly $\mathcal{C}$-hypersimple set is $\mathcal{C}$-simple.

In late 1970s, Selman [20] introduced the notion of P-selective sets, which are analogues of semi-recursive sets in recursion theory. These sets connects P-immunity to P-hyperimmunity. In general, for any class $\mathcal{F}$ of total functions, we say that a set $S$ is $\mathcal{F}$-selective if there exists a function (called the selector) $f$ in $\mathcal{F}$ such that, for all pairs $(x, y) \in \Sigma^{*} \times \Sigma^{*}$, (i) $f(x, y) \in\{x, y\}$ and (ii) $\{x, y\} \cap S \neq \emptyset$ implies $f(x, y) \in S$. Recall the partial function class $\Sigma_{k}^{\mathrm{P}} \mathrm{SV}$. We use the notation $\Sigma_{k}^{P_{S}} \mathbf{S V}_{t}$ to denote the collection of all total functions in $\Sigma_{k}^{P} \mathbf{S V}$.

LEMMA 21 Let $k \in \mathbb{N}^{+}$. Every $\boldsymbol{\Sigma}_{k}^{\mathrm{P}}$-immune $\boldsymbol{\Sigma}_{k}^{\mathrm{P}} \mathbf{S V}_{\boldsymbol{t}}$-selective set is honestly $\boldsymbol{\Sigma}_{k}^{\mathrm{P}}$ hyperimmune. 
We give the proof of Lemma 21. Assume that $S$ is $\Sigma_{k}^{\mathrm{P}} \mathrm{SV}_{\boldsymbol{t}}$-selective via a selector $f$ and has a honest $\Sigma_{k}^{\mathrm{P}}$-array $\mathcal{D}=\left\{D_{\theta}\right\}_{s \in \Sigma^{*}}$ intersecting $S$ via a supporting function $g$. For each $y \in \operatorname{dom}(g)$, assuming $D_{y}=\left\{x_{1}, x_{2}, \ldots, x_{m}\right\}$ with $x_{1}<x_{2}<\cdots<x_{m}$, let $y_{1}=x_{1}$ and $y_{i+1}=f\left(y_{i}, x_{i+1}\right)$ for every $i \in\{1,2, \ldots, m-1\}$ and then define $h(y)=y_{m}$. This $h$ is honest and in $\Sigma_{k}^{\mathrm{P} S V}$. The set $B=\{x \mid \exists y[y \in \operatorname{dom}(h) \wedge h(y)=$ $x]\}$ is therefore infinite and in $\Sigma_{k}^{P}$ since $\operatorname{dom}(f)$ is infinite and $h$ is honest. Because $B \subseteq S, B$ cannot be $\Sigma_{k}^{P}$-immune. Note that our proof relativizes.

Observe that the complement of a $\Sigma_{k}^{\mathrm{P}} \mathbf{S} \mathbf{V}_{\boldsymbol{t}}$-selective set $S$ is also $\Sigma_{k}^{\mathrm{P}} \mathbf{S V} \mathbf{V}_{t}$-selective because the exchange of the output string of any selector for $S$ gives rise to a selector for $\overline{\boldsymbol{S}}$. It also follows from Lemma 21 that every NP-simple P-selective set is honestly NP-hypersimple since the complement of any P-selective set is also P-selective.

Next, we show that strong P-immunity does not imply honest P-hyperimmunity within the class E. Earlier, Balcázar and Schöning [2] created a strongly P-bi-immune set $S$ in $\mathrm{E}$ with the density $\left|S \cap \Sigma^{\leq n}\right|=2^{n+1}-n-1$ for all $n \in \mathbb{N}$. For each $x$, let $D_{x}$ consist of the first $|x|+1$ elements of $\Sigma^{|x|}$. Clearly, $D_{x}$ intersects $S$. This implies that $S$ is not honestly P-hyperimmune. Therefore, we obtain the following proposition.

PROPOSITION 22 There exists a strongly P-bi-immune set in E that is not honestly P-hyperimmune.

As a main theorem, we show the P-T-incompleteness of $\Sigma_{k}^{\mathrm{P}}$-hypersimple sets. Generally, we say that $A$ is $\Delta_{k}^{\mathrm{P}}$-T-reducible to $B$ if there exists an oracle $\Delta_{k}^{\mathrm{P}}$-machine $M$ which recognizes $A$ with access to $B$ as an oracle. If in addition $M$ on input $x$ makes only queries $y$ to $B$ that satisfy $|x| \leq p(|y|)$, where $p$ is a fixed polynomial, then we say that $A$ is $h-\Delta_{k}^{\mathrm{P}}-T$-reducible to $B$ via $M$. This reduction machine $M$ is simply called honest.

\section{THEOREM 23 Let $k \in \mathbb{N}^{+}$.}

1 No $\Sigma_{k}^{P}$-hypersimple set is $P$-T-complete for $\Sigma_{k}^{P}$.

2 No honestly $\Sigma_{k}^{\mathrm{P}}$-hypersimple set is h-P-T-complete for $\Sigma_{k}^{\mathrm{P}}$.

Note that it is not clear if we can replace the P-T-completeness in Theorem 23 by the $\Delta_{k}^{\mathbf{P}}$-T-completeness. Theorem 23 follows from Lemma 24 in the following fashion. We prove only the first claim. Assume that $B$ is a $\Sigma_{k}^{\mathrm{P}}$-hypersimple set that is P-T-complete for $\Sigma_{k}^{\mathrm{P}}$. Thus, $\Delta_{k}^{\mathrm{P}} \neq \Sigma_{k}^{\mathrm{P}}$. Clearly, $B$ is in EXP and every $\Sigma_{k}^{\mathrm{P}}$. set is P-T-reducible to $B$. By Lemma 24 , every $\Sigma_{k}^{P}$-set is almost $\Delta_{k}^{\mathrm{P}}$-immune. This contradicts Lemma 13. Therefore, $B$ cannot be $\Sigma_{k}^{\mathbf{P}}$-hypersimple.

LEMMA 24 Let $k \in \mathbb{N}^{+}$and let $A$ be any infinite set in $\Sigma_{k}^{P}$.

1 If $A$ is $P$-T-reducible to a $\Sigma_{k}^{\mathrm{P}}$-hyperimmune set in EXP, then $A$ is almost $\Delta_{k}^{\mathrm{P}}$ immune.

2 If $A$ is $h$-P-T-reducible to a honestly $\Sigma_{k}^{\mathrm{P}}$-hyperimmune set, then $A$ is almost $\Delta_{k}^{\mathrm{P}}$-immune.

Lemma 24 needs a key idea of Agrawal (mentioned earlier), who showed that no NP-simple set is h-P-btt-complete for NP. We extend his core argument to Lemma 
25. For convenience, a complexity class $\mathcal{C}$ is said to be closed under intersection with $\Delta_{k}^{\mathrm{P}}$-sets if, for any set $A$ in $\mathcal{C}$ and any set $B$ in $\Delta_{k}^{\mathrm{P}}$, the intersection $A \cap B$ is in $\mathcal{C}$.

LEMMA 25 Let $\mathcal{C}$ be any complexity class containing $\Delta_{k}^{P}$ such that $\mathcal{C}$ is closed under intersection with $\Delta_{k}^{P}$-sets. Let $A$ be any $\Delta_{k}^{P}$-levelable set in $\mathcal{C}$. If $A$ is $\Delta_{k}^{P}$-T-reducible to $B$ via a reduction machine $M$, then there exists an infinite set $C$ in $\mathcal{C}$ such that $Q(M, B, x) \cap B \neq \emptyset$ for all $x \in C$.

Bruschi [4] demonstrated how to construct a recursive oracle relative to which a $\Sigma_{k}^{\mathrm{P}}$-simple set exists. We can easily modify his proof to obtain a P-selective set that is $\Sigma_{k}^{P}$-simple in a relativized world. Since Lemma 21 relativizes, we obtain the following proposition.

PROPOSITION 26 For each $k \in \mathbb{N}^{+}$, there exists a recursive oracle $A$ such that a $\Sigma_{k}^{\mathrm{P}}(A)$-hypersimple set exists.

As Schaefer and Fenner [19] demonstrated, it is relatively easy to prove the existence of an honest $\mathrm{NP}^{G}$-hypersimple set relative to a generic oracle $G$. By contrast, Vereshchagin [24] proved the existence of an NP-simple set relative to a random oracle with probability 1 . Again, we modify his proof to construct a relativized P-selective NP-simple set. From Lemma 21, the next proposition follows.

PROPOSITION 27 With probability 1, an honestly $\mathrm{NP}^{X}$-hypersimple set exists relative to a random oracle $X$.

An important open problem is to prove that, at each level $k$ of the polynomialtime hierarchy, honest $\Sigma_{k}^{\mathrm{P}}$-hypersimple sets exist relative to a random oracle with probability 1 .

\section{Completeness Under Non-Honest Reductions}

Immunity has a deep connection to various completeness notions. For example, there is a simple, tt-complete set; however, no simple set is btt-complete. In the previous sections, we have shown that various types of resource-bounded simple sets cannot be complete under certain polynomial-time honest reductions. This section instead focuses on the incompleteness of simple sets under non-honest reductions.

To remove the honesty condition from reductions, we often need to make extra assumptions for similar incompleteness results. In mid 1980s, Hartmanis, $\mathrm{Li}$, and Yesha [6] proved that (i) no NP-immune set in EXP is P-m-hard for NP if NP $\nsubseteq$ SUBEXP and (ii) no NP-simple set is P-m-complete if NP $\cap$ co-NP $\nsubseteq$ SUBEXP. These results can be expanded to any $\Delta$-level of the polynomial-time hierarchy and of the subexponential-time hierarchy ${ }^{8}$. We also improve the latter claim.

\footnotetext{
${ }^{8}$ The $\Delta$-level of the subexponential-time hierarchy is defined as: SUB $\Delta_{0}^{\text {EXP }}=$ SUBEXP and SUB $\Delta_{k+1}^{\operatorname{EXP}}=\operatorname{SUBEXP}^{\Sigma_{k}^{\mathrm{P}}}$ for every $k \in \mathbb{N}$, where $\operatorname{SUBEXP}^{\boldsymbol{A}}$ denotes $\bigcap_{e}>0 \operatorname{DTIME}^{\boldsymbol{A}}\left(2^{n^{\boldsymbol{t}}}\right)$ for any oracle $A$. When $\boldsymbol{A}=\boldsymbol{\emptyset}$, we simply write SUBEXP for $\operatorname{SUBEXP}^{\boldsymbol{A}}$.
} 
To describe our expansion, we need the unambiguous complexity class $\mathbf{U}\left(\boldsymbol{\Sigma}_{k}^{\mathrm{P}} \cap \Pi_{k}^{\mathrm{P}}\right)$ introduced by Yamakami [25]. For any complexity class $\mathcal{C}$, a set $A$ is in $U(\mathcal{C})($ or $U \mathcal{C})$ if there exists a single-valued partial function $f$ such that (i) $f$ is polynomially bounded, (ii) $\operatorname{Graph}(f) \in \mathcal{C}$, and (iii) $A=\operatorname{dom}(f)$ [25].

\section{PROPOSITION 28 Let $\boldsymbol{j}$ and $\boldsymbol{k}$ be any nonnegative integers.}

1 No $\Sigma_{k}^{\mathrm{P}}$-immune set in $\Delta_{j}^{\mathrm{EXP}}$ is $\Delta_{k}^{\mathrm{P}}$-m-hard for $\boldsymbol{\Sigma}_{k}^{\mathrm{P}}$ if $\Sigma_{k}^{\mathrm{P}} \nsubseteq \mathrm{SUB} \Delta_{\max \{j, k\}}^{\mathrm{EXP}}$.

2 No $\boldsymbol{\Sigma}_{k}^{\mathrm{P}}$-simple set is $\Delta_{k}^{\mathrm{P}}$-m-complete for $\boldsymbol{\Sigma}_{k}^{\mathrm{P}}$ if $\mathrm{U}\left(\boldsymbol{\Sigma}_{k}^{\mathrm{P}} \cap \Pi_{k}^{\mathrm{P}}\right) \nsubseteq \mathrm{SUB} \Delta_{k}^{\mathrm{EXP}}$.

Note that Proposition 28(2) directly follows from Theorem 29(2).

The original result of Hartmanis et al. refers to the P-m-incompleteness of NPsimple sets. Recently, Schaefer and Fenner [19] showed a similar result for the P$1 \mathrm{tt}$-completeness. They proved that no NP-simple set is P-1tt-complete for NP if UP $\nsubseteq$ SUBEXP. A key to their proof is the fact ${ }^{9}$ that $\operatorname{Sep}$ (SUBEXP, NP) implies UP $\subseteq$ SUBEXP, where $\operatorname{Sep}(\mathcal{C}, \mathcal{D})$ means the separation property in [25] that, for any two disjoint sets $A, B \in \mathcal{D}$, there exists a set $S \in \mathcal{C} \cap$ co-C satisfying that $A \subseteq S \subseteq \bar{B}$.

The following theorem shows that the assumption UP $\nsubseteq$ SUBEXP in [19] can be replaced by $\mathrm{U}(\mathrm{NP} \cap$ co-NP) $\nsubseteq \mathrm{SUBEXP}$.

THEOREM 29 Let $j, k \in \mathbb{N}^{+}$.

1 No $\Sigma_{k}^{\mathrm{P}}$-immune set in $\Delta_{j}^{\mathrm{EXP}}$ is $\Delta_{k}^{\mathrm{P}}$-ltt-hard for $\mathrm{U}\left(\Sigma_{k}^{\mathrm{P}} \cap \Pi_{k}^{\mathrm{P}}\right)$ if $\mathrm{U}\left(\Sigma_{k}^{\mathrm{P}} \cap \Pi_{k}^{\mathrm{P}}\right) \notin$ $\mathrm{SUB} \Delta_{\max \{j, k\}}^{\operatorname{EXP}}$.

2 No $\Sigma_{k}^{\mathrm{P}}$-simple set is $\Delta_{k}^{\mathrm{P}}$-Itt-complete for $\Sigma_{k}^{\mathrm{P}}$ if $\mathrm{U}\left(\Sigma_{k}^{\mathrm{P}} \cap \Pi_{k}^{\mathrm{P}}\right) \nsubseteq \mathrm{SUB} \Delta_{k}^{\mathrm{EXP}}$.

Theorem 29 follows from the technical lemmas: Lemmas 30 and 31. The proof for its second claim proceeds as follows. Assume that $B$ is $\Delta_{k}^{P}-1$ tt-complete for $\Sigma_{k}^{P}$. Choose an infinite set $A \in \mathrm{U}\left(\Sigma_{k}^{\mathrm{P}} \cap \Pi_{k}^{\mathrm{P}}\right)-\mathrm{SUB} \Delta_{k}^{\mathrm{EXP}}$, which is of the form $\{x \mid$ $\exists y[\langle\boldsymbol{x}, y\rangle \in \operatorname{Graph}(\boldsymbol{f})]\}$ for a certain polynomially-bounded partial function $f$ whose graph is in $\Sigma_{k}^{\mathrm{P}} \cap \Pi_{k}^{\mathrm{P}}$. Similar to [25], set $A_{1}=\{\langle x, z\rangle \mid \exists y[z \leq y \wedge\langle x, y\rangle \epsilon$ $\operatorname{Graph}(f)]\}$ and $A_{2}=\{\langle x, z\rangle \mid \exists y[z<y \wedge\langle x, y\rangle \in G \operatorname{Gaph}(f)]\}$. Clearly, $A_{1}$ and $A_{2}$ are in $\mathrm{U}\left(\Sigma_{k}^{\mathrm{P}} \cap \Pi_{k}^{\mathrm{P}}\right)-\mathrm{SUB} \Delta_{k}^{\mathbf{E X P}}$. Since $A_{1}$ is $\Delta_{k}^{\mathrm{P}}-1$ tt-reducible to $B$, by Lemma 30, there exists a set $C \in \Delta_{k}^{\mathrm{P}}$ such that $A_{1} \cap C$ is infinite and coinfinite and $A_{1} \cap C$ is h- $\Delta_{k}^{\mathrm{P}}$-1tt-reducible to $B$. Applying Lemma 31(1), we obtain a set $D \in \Delta_{k}^{\mathrm{P}}$ and a total F $\Delta_{k}^{P}$-function $f$ such that $\overline{A_{1} \cap C} \cap \bar{D}$ is finite. $f \Delta_{k}^{P}$-m-reduces $A_{1} \cap C$ to $\bar{B}$, and $f$ is honest on the domain $D$. Since $A_{2} \subseteq \overline{A_{1} \cap C}, f\left(A_{2}\right) \subseteq \widetilde{B}$. Moreover, $A_{2} \cap D$ is infinite. The honesty of $f$ on $D$ implies that $\bar{B}$ has an infinite $\Sigma_{k}^{\mathrm{P}}$-subset $f\left(A_{2} \cap D\right)$.

The key idea of Hartmanis et al. [6] is to find a set that can be honestly reducible. Lemma 30 is a " $1 \mathrm{tt}$ " version of a technical part of [6].

LEMMA 30 Let $j, k \in \mathbb{N}^{+}$. Assume that $A \notin \mathrm{SUB} \Delta_{\max \{j, k\}}^{\operatorname{EXP}}$ and $B \in \Delta_{j}^{\mathrm{EXP}}$. If $A$ is $\Delta_{k}^{\mathrm{P}}$-ltt-reducible to $B$, then there exists a set $C$ in $\Delta_{k}^{\mathrm{P}}$ such that (i) $A \cap C$ is $h-\Delta_{k}^{P}-1$ tt-reducible to $B$ and (ii) $A \cap C$ and $\bar{A} \cap C$ are infinite and coinfinite.

${ }^{9}$ Actually, the result of Schaefer and Fenner can be strengthened in the following way: Sep(SUBEXP, U(NP $\cap$ co-NP)) if and only if U(NP $\cap$ co-NP) $\subseteq$ SUBEXP. This is obtained by analyzing a similar result in [25]. 
LeMMA 31 Let $k \in \mathbb{N}^{+}$and $A, B \subseteq \Sigma^{*}$. Assume that $B$ is $\boldsymbol{\Sigma}_{k}^{P}$-immune and $A$ is $h-\Delta_{k}^{P}-1$ tt-reducible to $B$.

1 If $A \in \Sigma_{k}^{\mathrm{P}}$, then there exist a set $D \in \Delta_{k}^{\mathrm{P}}$ and a total function $f \in \mathrm{F} \Delta_{k}^{\mathrm{P}}$ such that $\bar{A} \cap \bar{D}$ is finite, $f \Delta_{k}^{\mathrm{P}}$-m-reduces $A$ to $\bar{B}$, and $f$ is honest on the domain $D$.

2 A belongs to $\Sigma_{k}^{\mathrm{P}} \cap \Pi_{k}^{\mathrm{P}}$ if and only if A belongs to $\Delta_{k}^{\mathrm{P}}$.

\section{Limited Immunity and Simplicity}

Within our current knowledge, we cannot prove or disprove the existence of an NPsimple set. The difficulty comes from the fact that an NP-immune set requires every NP-subset to be finite. If we restrict our attention to certain types of NP-subsets, then we may overcome the difficulty. Under the name of $k$-immune sets, Homer [7] required only $\mathbf{N P}_{(k)}$-subsets, for a fixed number $k$, to be finite. He then demonstrated how to construct a $\boldsymbol{k}$-simple set within NP using Ladner's delayed diagonalization technique.

In this section, we investigate the notions obtained by restricting the requirements for immunity and simplicity. We first review Homer's notions of $k$-immunity and $k$-simplicity.

DEFINITION 32 Let $\boldsymbol{k}$ be any number in $\mathbf{N}^{+}$.

1 A set $S$ is $\boldsymbol{k}$-immune if $S$ is infinite and there is no index $\boldsymbol{i}$ in $I N D E X_{(\boldsymbol{k})}$ such that $\boldsymbol{W}_{\boldsymbol{i}}$ is infinite and $\boldsymbol{W}_{\boldsymbol{i}} \subseteq S$.

2 A set $S$ is $\boldsymbol{k}$-simple if $S$ belongs to NP and $\overline{\boldsymbol{S}}$ is $\boldsymbol{k}$-immune.

An "effective" version of immune and simple sets, called effectively immune and effectively simple sets, has been studied in recursion theory. Effectively simple sets are known to be T-complete and there also exists an effectively simple tt-complete set. If $A$ is strongly effectively immune, then $\bar{A}$ cannot be immune. Analogously, we consider a resource-bounded version of such effectively immune and simple sets. Here, we freely identify binary strings with natural numbers using the lexicographic order on $\boldsymbol{\Sigma}^{*}$.

\section{DEFINITION 33 Let $k \in \mathbb{N}^{+}$.}

1 A set $S$ is feasibly $k$-immune if(i) $S$ is infinite and (ii) there exists a polynomial $p$ such that, for every index $\boldsymbol{i}$ in INDEX $\boldsymbol{X}_{(\boldsymbol{k})}, W_{\mathbf{i}} \subseteq S$ implies $\left|\boldsymbol{W}_{\mathbf{i}}\right| \leq 2^{\boldsymbol{p}^{(\mathbf{i})}}$.

2 A set $S$ is feasibly $\boldsymbol{k}$-simple if $\mathrm{S}$ is in NP and $\overline{\boldsymbol{S}}$ is feasibly $\boldsymbol{k}$-immune.

We can easily prove the existence of a feasibly $k$-immune set in $\Delta_{2}^{P}$ for each $k \in \mathbb{N}^{+}$. From Definition 33, every feasibly $k$-simple set is $k$-simple. The converse, however, does not hold since there exists a $\boldsymbol{k}$-simple set which is not feasibly $\boldsymbol{k}$-simple for each number $\boldsymbol{k}$ in $\mathbb{N}^{+}$. The theorem below is slightly stronger than this claim since any feasibly $k$-simple set is also feasibly 1 -simple.

THEOREM 34 For each $\boldsymbol{k} \in \mathbb{N}^{+}$, there exists a $\boldsymbol{k}$-simple set which is not feasibly 1-simple. 
We return to the old question of whether NP-simple sets exist. There seems no strong evidence that suggests the existence of such a set. Only relativization provides a world where NP-simple sets exist. At the same time, we can also construct another world where these sets do not exist. These relativization results clearly indicate that the question of whether NP-simple sets exist needs unrelativizable proof techniques.

In the past few decades, the Berman-Hartmanis isomorphism conjecture has served as a working hypothesis in connection to NP-complete problems. By contrast, there has been no "natural" working hypothesis that yields the existence of NP-simple sets. For example, the hypothesis $\mathbf{P} \neq \mathbf{N P}$ does not suffice since Homer and Maass [8] showed a relativized world where the assumption $\mathrm{P} \neq \mathrm{NP}$ does not imply the existence of an NP-simple set. Motivated by Homer's $k$-simplicity result, we propose the following working hypothesis:

- The $k$-immune hypothesis: There exists a positive integer $k$ such that every infinite NP set has an infinite $\mathrm{NP}_{(\boldsymbol{k})}$-subset.

Under this hypothesis, we can derive the desired consequence: the existence of NPsimple sets.

LEMMA 35 If the $\mathrm{k}$-immune hypothesis holds, then there exists an NP-simple set.

Assume that the $k$-immune hypothesis is true; that is, there exists a positive integer $k$ such that every infinite NP-set has an infinite $\mathrm{NP}_{(k)}$-subset. Consider any $k$-simple set $A$. We claim that $A$ is NP-simple. If $A$ is not NP-simple, then $\bar{A}$ has an infinite NP-subset $B$. By our assumption, $B$ contains an infinite $\mathrm{NP}_{(k)}$-subset. Hence, $A$ cannot be $k$-simple, a contradiction. Therefore, $A$ is NP-simple.

To close this section, we claim the following result concerning the $k$-immune hypothesis. The proof uses weak forcing.

PROPOSITION 36 The $k$-immune hypothesis fails relative to a generic oracle.

Final Note. All the proofs that are omitted from this extended abstract will appear in its forthcoming complete version.

\section{References}

[1] J. L. Balcázar, Simplicity, relativizations, and nondeterminism, SIAM J. Comput. 14 (1985) 148-157.

[2] J. L. Balcázar and U. Schöning, Bi-immune sets for complexity classes, Math. Systems Theory 18 (1985) 1-10.

[3] C. H. Bennett and J. Gill, Relative to a random oracle $A, \mathbf{P}^{A} \neq \mathbf{N P}^{A} \neq$ co-NP $^{A}$ with probability 1, SIAMJ. Comput. 10 (1981) 96-113.

[4] D. Bruschi, Strong separations of the polynomial hierarchy with oracles: constructive separations by immune and simple sets, Theoret. Comput. Sci. 102 (1992) 215-252. 
[5] P. Flajolet and J. M. Steyaert, On sets having only hard subsets, in Proc. 2nd Intern. Colloq. on Automata, Languages, and Programming, LNCS, Springer, Vol.14, pp.446457,1974.

[6] J. Hartmanis, M. Li, and Y. Yesha, Containment, separation, complete sets, and immunity of complexity classes, in: Proc. 13th Intern. Colloq. on Automata, Languages, and Programming, LNCS, Springer, Vol.226, pp. 136-145, 1986.

[7] S. Homer, On simple and creative sets in NP, Theoret. Comput. Sci., 47 (1986) 169-180.

[8] S. Homer and W. Maass, Oracle-dependent properties of the lattice of NP sets, Theoret. Comput. Sci., 24 (1983) 279-289.

[9] D. Joseph and P. Young, Some remarks on witness functions for nonpolynomial and noncomplete sets in NP, Theoret. Comput. Sci. 39 (1985) 225-237.

[10] K. Ko, Nonlevelable sets and immune sets in the accepting density hierarchy in NP, Math. Systems Theory 18 (1985) 189-205.

[11] K. Ko and D. Moore, Completeness, approximation and density, SIAM J. Comput. 10 (1981) 787-796.

[12] A. Meyer and L. Stockmeyer, The equivalence problem for regular expressions with squaring requires exponential time, in Proc. 13th IEEE Symp. on Switching andAutomata theory, pp.125-129, 1973.

[13] N. Lynch, On reducibility to complex or sparse sets, Journal of ACM, 22 (1975) 341-345.

[14] P. Orponen, A classification of complexity core lattices, Theoret. Comput. Sci., 47 (1986) 121-130.

[15] P. Orponen, K. Ko, U. Schöning, and O. Watanabe, Instance complexity, Journal of ACM, 41 (1994) 96-121.

[16] P. Orponen, D. Russo, and U. Schöning, Optimal approximations and polynomially levelable sets, SIAM J. Comput., 15 (1986) 399-408.

[17] E. L. Post, Recursively enumerable sets of positive integers and their decision problems, Bull. Am. Math. Soc. 50 (1944) 284-316.

[18] D. A. Russo, Optimal approximations of complete sets, in Proc. 1st Annual Conference on Structure in Complexity Theory, LNCS, Springer, Vol.223, pp.311-324, 1986.

[19] M. Schaefer and S. Fenner, Simplicity and strong reductions, manuscript, 2000.

[20] A. Selman, P-selective sets, tally languages and the behavior of polynomial time reducibilities on NP, Math. Systems Theory, 13 (1979) 55-65.

[21] L. Torenvliet, A second step toward the strong polynomial-time hierarchy, Math. Systems Theory 21 (1988) 99-123.

[22] L. Torenvliet and P. van Emde Boas, Simplicity, immunity, relativization and nondeterminism, Inform. and Comput. 80 (1989) 1-17.

[23] V. A. Uspenskii, Some remarks on r.e. sets, Zeit. Math. Log. Grund. Math. 3 (1957) 157170.

[24] N. K. Vereshchagin, Relationships between NP-sets, CoNP-sets and P-sets relative to random oracles, in Proc. 8th IEEE Conf. on Structure in Complexity Theory, pp.132-138, 1993.

[25] T. Yamakami, Structural properties for feasibly computable classes of type two, Math. Systems Theory 25 (1992) 177-201.

[26] T. Yamakami, Simplicity, unpublished manuscript, University of Toronto, 1995. 